\title{
Atiyah-Manton construction of Skyrmions in eight dimensions
}

\author{
Atsushi Nakamula, Shin Sasaki and Koki Takesue \\ Department of Physics, Kitasato University, \\ Sagamihara 252-0373, Japan \\ E-mail: nakamula@sci.kitasato-u.ac.jp, shin-s@kitasato-u.ac.jp, \\ ktakesue@sci.kitasato-u.ac.jp
}

ABSTRACT: We show that the eight-dimensional instanton solution, which satisfies the self-duality equation $F \wedge F=*_{8} F \wedge F$, realizes the static Skyrmion configuration in eight dimensions through the Atiyah-Manton construction. The relevant energy functional of the Skyrme field is obtained by the formalism developed by Sutcliffe. By comparing the Skyrmion solution associated with the extreme of the energy, with the Atiyah-Manton solution constructed by the instantons, we find that they agree with high accuracy. This is a higher-dimensional analogue of the Atiyah-Manton construction of Skyrmions in four dimensions. Our result indicates that the instanton/Skyrmion correspondence seems to be an universal property in $4 k(k=1,2, \ldots)$ dimensions.

Keywords: Field Theories in Higher Dimensions, Solitons Monopoles and Instantons, Topological Field Theories

ArXiv EPRINT: 1612.06957 


\section{Contents}

1 Introduction 1

2 Eight-dimensional Skyrme model from quartic Yang-Mills theory 3

2.1 Overview of the Sutcliffe's truncation in four dimensions 3

$\begin{array}{lll}2.2 & \text { Eight-dimensional Skyrme model } & 6\end{array}$

3 Eight-dimensional Skyrmions from instantons $\quad 7$

4 Higher dimensional generalization $\quad 12$

5 Conclusion and discussions $\quad 14$

A Derivation of the eight-dimensional Skyrme model with the hedgehog ansatz

\section{Introduction}

The Skyrme model [1] is a model for pions in the low-energy effective theory of QCD. The model is a four-dimensional non-linear sigma model whose target space is $S^{3} \sim \mathrm{SU}(2)$, and composed of the fourth order derivative term in addition to the canonical kinetic term. The fourth order derivative term guarantees the stability of solitons of co-dimension three, which are called Skyrmions. The Skyrmions are characterized by the homotopy class $\pi_{3}(\mathrm{SU}(2))=\mathbb{Z}$ and they are regarded as Baryons. The energy functional of the Skyrme model has the Bogomol'nyi bound given by the topological charge associated with the homotopy. This topological charge is identified with the Baryon number. However, no analytic solutions that saturate the lower bound of the energy have been found so far. ${ }^{1}$ There have only been obtained the numerical solutions of Skyrmions, which indeed exceed the energy bound. This reflects the fact that the original four dimensional Skyrme model does not have the BPS property.

Finding proper solutions of Skyrmions is a long standing problem. There are several directions to construct solutions. For example, the rational map ansatz provides a good approximation to the Skyrmion solutions [5]. This includes solutions corresponding to higher Baryon numbers. Although they can not saturate the energy bound, the rational map solutions have close energies to the normalized Baryon charges. Alternatively, there is another promising approach to Skyrmions known as the Atiyah-Manton construction [6]. Atiyah and Manton pointed out that the holonomy of the Yang-Mills instantons in the fourdimensional Euclid space ${ }^{2}$ gives a well approximated static Skyrmion solutions. Although,

\footnotetext{
${ }^{1}$ This is not the case for Skyrme models in curved spaces. For example, see [2-4] and references therein.

${ }^{2}$ The case for the curved spaces was discussed in [7].
} 
the origin of this approximation is not transparent, a physical interpretation to the AtiyahManton construction of Skyrmions was discussed in [8, 9].

Even though the Skyrmion solutions are well-approximated by instantons, they never saturate the Bogomol'nyi bound of the energy. In order to understand the obscure connection between the Yang-Mills instantons and Skyrmions, we need further penetrating analysis. In this context, in [10], inspired by a holographic QCD model [11], it is proposed a systematic derivation of the energy functional for the static Skyrme field from the Yang-Mills action in four dimensions. In the derivation, the introduction of the tower of mesons originated from the Kaluza-Klein-like expansion modes in higher dimensions makes the Atiyah-Manton solution have closer energy to the bound [12]. Therefore, including the higher expansion modes in the Atiyah-Manton solution leads to the better approximation to the Skyrmions. Moreover, this relation is generalized to lower dimensions. For example, an analogue of the Atiyah-Manton construction in two dimensions is proposed [13, 14] where the sine-Gordon soliton solution in one dimensions is well-approximated by the $\mathbb{C} P^{1}$ lump - the two-dimensional instantons. These facts remarkably suggest that there is a deep correspondence between instantons or solitons and Skyrmion-like objects in various dimensions.

The instantons in four dimensions satisfy the self-duality equation $F=*_{4} F$. Here $F$ is the field strength 2 -form of the gauge field and $*_{d}$ is the Hodge dual operator in $d$ dimensions. A natural higher-dimensional generalization of instantons is a solution to the self-duality equations in $d=4 k$ dimensions $F^{k}={ }_{*}{ }_{4 k} F^{k}$ where $F^{k}$ is the $k$ wedge products of $F$. The $k=1$ case corresponds to the ordinary instantons in four-dimensions while the $k \geq 2$ cases are their generalization. The first non-trivial example is the $k=2$ case, namely, the self-dual instantons in eight dimensions. This was studied so far from various viewpoints $[15,16]$. On the other hand, it is possible to consider higher-dimensional generalizations of Skyrmions [17].

In this paper we study the relation between instantons and Skyrmions in higher dimensions. In particular, we focus on the eight-dimensional self-dual instantons that satisfy $F \wedge F=*_{8} F \wedge F$. The self-duality relation is obtained by the Bogomol'nyi completion of the quartic Yang-Mills action in eight dimensions. We will derive the energy functional for the static Skyrme field from the quartic Yang-Mills action by the reduction procedure developed by Sutcliffe [10]. The Derrick's theorem indicates that the model admits static soliton solutions which we call the eight-dimensional Skyrmions. We will find the numerical solution of the above mentioned Skyrmion. We will then calculate a field configuration through the Atiyah-Manton construction applied to the eight-dimensional instanton and find that this gives a good approximation to the numerical solution of the Skyrmion. Our results strongly suggest that the instanton/Skyrmion correspondence holds even in $4 k$ dimensions and this relation is an universal property.

The organization of this paper is as follows. In section 2, we give a brief overview of the prescription by Sutcliffe in four dimensions. We then derive the energy functional for the static Skyrme field in eight dimensions from the quartic Yang-Mills action. In section 3, we perform the numerical analysis to solve the equation of motion. We find a spherically symmetric solution to the Skyrmions in eight dimensions. We then calculate 
the holonomy associated with the self-dual instanton in eight dimensions and construct the Atiyah-Manton solution. We will show a good agreement between the numerical solution and the Atiyah-Manton solution. Section 4 is an analysis of higher dimensional generalizations. Section 5 is devoted to the conclusion and discussions. The detail derivation of the energy functional for the static Skyrme field with hedgehog ansatz in eight dimensions is shown in appendix.

\section{Eight-dimensional Skyrme model from quartic Yang-Mills theory}

In this section we introduce a Skyrme model in eight dimensions following the formalism developed by Sutcliffe [10]. Before going to the eight-dimensional analysis, we give an overview of the derivation for the ordinary Skyrme model in four dimensions.

\subsection{Overview of the Sutcliffe's truncation in four dimensions}

The four-dimensional energy functional for static fields ${ }^{3}$ of the Skyrme model is obtained by a reduction of the usual quadratic Yang-Mills action in the four-dimensional Euclidean space. The action is

$$
S=-\frac{1}{2 \kappa g^{2}} \int \operatorname{Tr}\left[*_{4} F \wedge F\right]=-\frac{1}{4 \kappa g^{2}} \int d^{4} x \operatorname{Tr}\left[F_{m n} F^{m n}\right] .
$$

Here $F=\frac{1}{2 !} F_{m n} d x^{m} \wedge d x^{n},(m, n=1, \ldots, 4)$ is the gauge field strength 2 -form. The component is given by $F_{m n}=\partial_{m} A_{n}-\partial_{n} A_{m}+\left[A_{m}, A_{n}\right]$. The gauge field $A_{m}$ is in the adjoint representation of a gauge group $G$ and it is expanded by the generators $T^{a}(a=$ $1, \ldots \operatorname{dim} \mathcal{G})$. Here $\mathcal{G}$ is the Lie algebra associated with $G$ and $\kappa$ is the normalization constant for the generators $\operatorname{Tr}\left[T^{a} T^{b}\right]=\kappa \delta^{a b}$. Here $g$ is the gauge coupling constant. Making the action (2.1) be the completely square form results in the Bogomol'nyi-PrasadSommerfield (BPS) self-duality equation $F=*_{4} F$ whose solutions are called instantons. Since the Yang-Mills action (2.1) has the scale invariance, instanton solutions that saturate the Bogomol'nyi bound have a size modulus.

It is proposed in [10] that a holography-inspired reduction of the four-dimensional Yang-Mills action (2.1) provides the energy functional for the static Skyrme field. Following the prescription in [10], we first decompose the four-dimensional Euclidean space into the three-dimensional physical space and a "fictious" direction: $x^{m}=\left(x^{i}, x^{4}\right)$ where $i=$ $1, \ldots, 3$. We then expand the four-dimensional gauge field $A_{m}\left(x^{i}, x^{4}\right)$ in the infinite line along the $x^{4}$-direction by a complete orthonormal basis with the square integrable function. A suitable basis with the boundary condition $A_{i}\left(x^{i}, x^{4}\right) \rightarrow 0$ as $x^{4} \rightarrow \infty$ is the Hermite function $\psi_{n}(z)=\frac{(-1)^{n}}{\sqrt{n ! 2^{n} \sqrt{\pi}}} e^{\frac{1}{2} z^{2}} \frac{d^{n}}{d z^{n}} e^{-z^{2}}$. Then we have an expansion,

$$
A_{m}\left(x^{i}, x^{4}\right)=\sum_{n=0}^{\infty} \mathcal{A}_{m}^{(n)}\left(x^{i}\right) \psi_{n}\left(x^{4}\right),
$$

\footnotetext{
${ }^{3}$ We sometimes call this the three-dimensional action in Euclid space.
} 
where $\mathcal{A}_{m}^{(n)}\left(x^{i}\right)$ are expansion coefficients, which will be determined later. Next, we perform the gauge transformation by which the component $A_{4}$ is set to be zero. By this gauge transformation, the components of the gauge field $A_{i}$ is transformed as

$$
A_{i} \longrightarrow \hat{g} A_{i} \hat{g}^{-1}+\hat{g} \partial_{i} \hat{g}^{-1}
$$

where the gauge parameter $\hat{g}$ is given by

$$
\hat{g}\left(x^{i}, x^{4}\right)=-\mathrm{P} \exp \left[\int_{-\infty}^{x^{4}} d \xi A_{4}\left(x^{i}, \xi\right)\right] .
$$

Here the symbol $\mathrm{P}$ stands for the path-ordering. The asymptotic behavior of the Hermite function $\psi_{n}(\infty)=0$ and the boundary condition $A_{i}\left(x^{i}, \infty\right)=0$ determines the gauge field $A_{i}\left(x^{i}, x^{4}\right)$ in the gauge $A_{4}=0$. This is given by [10],

$$
A_{i}\left(x^{i}, x^{4}\right)=u_{i}\left(x^{i}\right) \psi_{+}\left(x^{4}\right)+\sum_{n=0}^{\infty} W_{i}^{n}\left(x^{i}\right) \psi_{n}\left(x^{4}\right),
$$

where $\psi_{+}(z)=\frac{1}{2}+\frac{1}{2} \operatorname{erf}(z / \sqrt{2})$ and the error function is defined by $\operatorname{erf}(z)=\frac{2}{\sqrt{\pi}} \int_{0}^{z} d \xi e^{-\xi^{2}}$. The gauge field is decomposed into the "zero-mode" $u\left(x^{i}\right)$ :

$$
u_{i}\left(x^{i}\right)=U \partial_{i} U^{-1}, \quad U\left(x^{i}\right)=\hat{g}\left(x^{i}, x^{4}=\infty\right),
$$

and the infinite tower of the vector fields $W_{i}^{n}\left(x^{i}\right)$. The zero-mode $u\left(x^{i}\right)$ is identified with the Skyrme field while the higher modes $W_{i}^{n}\left(x^{i}\right)$ can be interpreted as "vector mesons". This analysis is completely parallel to the Kaluza-Klein reduction in which a field is expanded by the Fourier modes $e^{i n x^{4} / 2 \pi R}$ along the compact circle $x^{4} \sim x^{4}+2 \pi R$. Note that the expansion along an infinite line enable us to realize the Skyrme field $U$ by the holonomy of the gauge field:

$$
U\left(x^{i}\right)=-\mathrm{P} \exp \left[\int_{-\infty}^{\infty} d x^{4} A_{4}\left(x^{i}, x^{4}\right)\right] .
$$

Although it is possible to compute $W_{i}^{n}$, let us focus on the leading approximation, i.e. we neglect all the vector meson modes and focus only on the Skyrme field $U\left(x^{i}\right)$. We call this the Sutcliffe's truncation. Then, in the gauge $A_{4}=0$, we have the following decomposition of the gauge field strength:

$$
\begin{aligned}
& F_{i 4}=U \partial_{i} U^{-1} \partial_{4} \psi_{+}\left(x^{4}\right)=R_{i} \frac{\psi_{0}\left(x^{4}\right)}{\sqrt{2} \pi^{\frac{1}{4}}}, \\
& F_{i j}=\left[R_{i}, R_{j}\right] \psi_{+}\left(x^{4}\right)\left(\psi_{+}\left(x^{4}\right)-1\right),
\end{aligned}
$$

where $R_{i}=U \partial_{i} U^{-1}$ is interpreted as the right current.

Now it is easy to show that the Sutcliffe's truncation of the Yang-Mills action (2.1) gives the energy functional for the static Skyrme field. Plugging the decomposition (2.8) into the quadratic Yang-Mills action (2.1) and performing the integration over $x^{4}$, then we find

$$
S=\frac{1}{\kappa g^{2}} \int d^{3} x\left(-\frac{c_{1}}{2} \operatorname{Tr}\left[R_{i} R_{i}\right]-\frac{c_{2}}{16} \operatorname{Tr}\left[R_{i}, R_{j}\right]^{2}\right)
$$




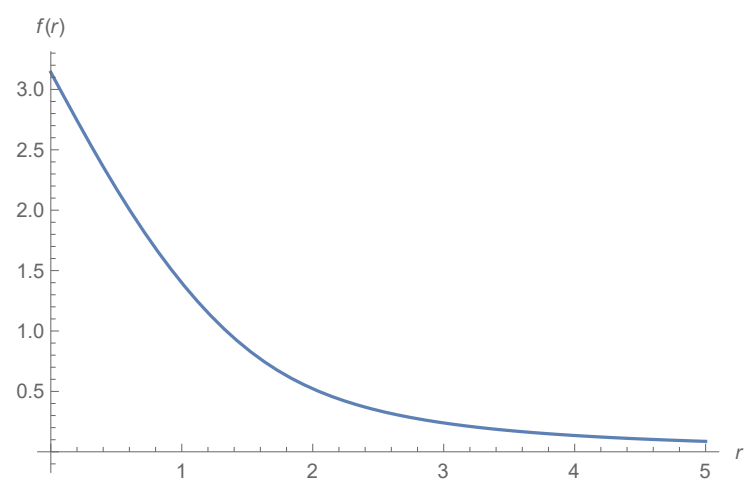

(a) The profile for $4 \mathrm{~d}$ Skyrmion.

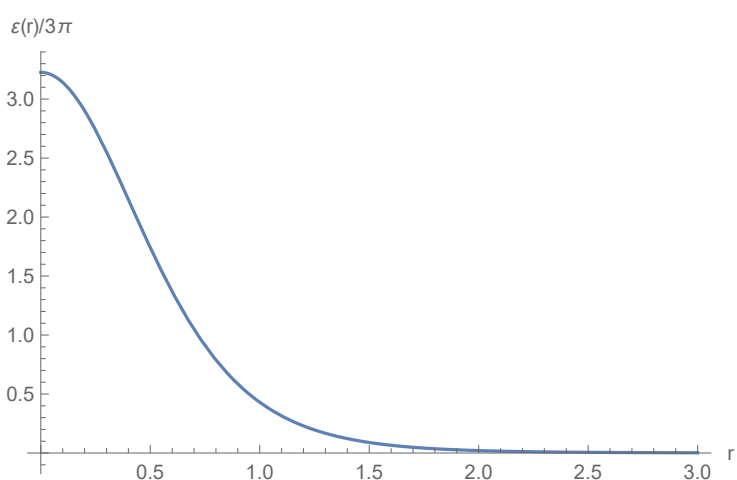

(b) The energy density plot.

Figure 1. The numerical profile for $f(r)$ and the plot for the energy density $\mathcal{E}(r)$.

where the numerical factors are calculated as $c_{1}=\frac{1}{4 \sqrt{\pi}} \simeq 0.141, c_{2}=2 \int_{-\infty}^{\infty} d x^{4} \psi_{+}^{2}\left(\psi_{+}-\right.$ $1)^{2} \simeq 0.198$. These numerical factors can be set to $c_{1}=c_{2}=1$ by the rescalings of the length $x^{i} \rightarrow \sqrt{c_{2} / c_{1}} x^{i}$ and the overall factor of the action $S \rightarrow \frac{1}{\sqrt{c_{1} c_{2}}} S$. We therefore consider the natural unit $c_{1}=c_{2}=1$ and set $\kappa=1, g=1$ for simplicity. After the rescaling, the action (2.9) becomes the energy functional for the static Skyrme field:

$$
E_{\text {Skyrme }}=\int d^{3} x\left(-\frac{1}{2} \operatorname{Tr}\left[R_{i} R_{i}\right]-\frac{1}{16} \operatorname{Tr}\left[R_{i}, R_{j}\right]^{2}\right) .
$$

The Bogomol'nyi completion of the energy functional (2.10) gives the energy bound $E_{\text {Skyrme }} \geq 12 \pi^{2}|B|$ where $B=-\frac{1}{24 \pi^{2}} \int d^{3} x \varepsilon_{i j k} \operatorname{Tr}\left[R_{i} R_{j} R_{k}\right]$ is the topological charge, namely, the Baryon number. Here $\varepsilon_{i j k}$ is the Levi-Civita symbol. The equation of motion derived from $(2.10)$ is

$$
\partial_{i}\left(R_{i}-\frac{1}{4}\left[R_{j},\left[R_{j}, R_{i}\right]\right]\right)=0
$$

No analytic solutions to this equation have been found but a spherically symmetric solution is dealt with the following hedgehog ansatz:

$$
U=\exp \left(i f(r) \hat{x}^{i} \tau_{i}\right)
$$

Here $\hat{x}^{i}=\frac{x^{i}}{r}, r^{2}=x^{i} x^{i}$ and $\tau^{i}$ are the Pauli matrices, namely, the quaternion basis. The energy functional for this ansatz is evaluated to be

$$
E_{\text {Skyrme }}=\int_{0}^{\infty} d r \int_{S^{2}} d \Omega_{2} \mathcal{E}(r)=2 \pi \int_{0}^{\infty} d r\left(r^{2}\left(\partial_{r} f\right)^{2}+2 \sin ^{2} f\left(1+\left(\partial_{r} f\right)^{2}\right)+\frac{\sin ^{4} f}{r^{2}}\right) .
$$

Here $\mathcal{E}(r)$ is the energy density and $d \Omega_{2}$ is the integral element of the two-dimensional sphere. The boundary condition is given by $f(0)=\pi, f(\infty)=0$. The numerical study is easily performed for this ansatz. The solution to the equation of motion (2.11) with the ansatz (2.12) is found in figure 1. The solution in figure 1 has the Baryon number $B=1$. 
We note that the energy functional (2.10) breaks the scale invariance presented in the Yang-Mills action. A physical origin of this violation comes from the Sutcliffe's truncation (2.8) where only the zero-mode (Skyrme field) is taken into account. Once we include all the vector meson modes $W_{i}^{n}$, the scale invariance is expected to be recovered.

\subsection{Eight-dimensional Skyrme model}

Now we generalize the procedure in the previous subsection to eight dimensions. In eight dimensions, the natural action whose BPS equation is the self-duality equation $F \wedge F=$ $*_{8}(F \wedge F)$ is that of the quartic Yang-Mills theory. The action is

$$
\begin{aligned}
S_{\text {quartic }} & =\frac{\alpha}{\kappa g^{2}} \int \operatorname{Tr}\left[*_{8}(F \wedge F) \wedge(F \wedge F)\right] \\
& =\left(\frac{1}{2 !}\right)^{4} \frac{4}{4 !} \frac{\alpha}{\kappa g^{2}} \int d^{8} x \operatorname{Tr}\left[\left(F^{M N} F^{P Q}\right)^{2}-4 F^{M N} F^{P Q} F_{M P} F_{N Q}+\left(F^{M N} F_{M N}\right)^{2}\right] .
\end{aligned}
$$

Here $M, N, \ldots=1, \ldots, 8$ and the component of the gauge field strength 2-form $F=$ $\frac{1}{2 !} F_{M N} d x^{M} \wedge d x^{N}$ is $F_{M N}=\partial_{M} A_{N}-\partial_{N} A_{M}+\left[A_{M}, A_{N}\right]$. A constant $\alpha$ has mass dimension $[\alpha]=-4$ and $g$ is the gauge coupling constant whose mass dimension is -2 . In the following, we set $\alpha / g^{2}=96$ and $\kappa=1$ for simplicity. The gauge field $A_{M}$ is in the adjoint representation of a Lie algebra associated with the gauge group $G$. We consider a gauge group $G$ which admits a non-trivial homotopy $\pi_{7}(G)=\mathbb{Z} .^{4}$

The analysis is completely parallel to the four-dimensional case. We decompose the directions $x^{M}=\left(x^{I}, x^{8}\right),(I=1, \ldots, 7)$ and expand the gauge field in terms of the Hermite function $\psi_{n}\left(x^{8}\right)$. The Sutcliffe's truncation provides the static Skyrme field in eight dimensions through the relations (2.8). Plugging the expansion (2.8) into the quartic Yang-Mills action (2.14) and performing the integration over the $x^{8}$-direction, we obtain the energy functional for the static Skyrme field:

$$
\begin{aligned}
E_{\text {Skyrme }}=\int d^{7} x \operatorname{Tr}[ & c_{2}\left(\left[R_{I}, R_{J}\right]\left[R_{I}, R_{J}\right]\right)^{2}+c_{2}\left(\left[R_{I}, R_{J}\right]\left[R_{K}, R_{L}\right]\right)^{2} \\
& -4 c_{2}\left[R_{I}, R_{J}\right]\left[R_{K}, R_{L}\right]\left[R_{I}, R_{K}\right]\left[R_{J}, R_{L}\right] \\
& +4 c_{1}\left(\left[R_{I}, R_{J}\right]\right)^{2} R_{K}^{2}+4 c_{1}\left(\left[R_{I}, R_{J}\right] R_{K}\right)^{2}-4 c_{1}\left[R_{I}, R_{J}\right] R_{K}\left[R_{I}, R_{K}\right] R_{J} \\
& \left.+8 c_{1}\left[R_{I}, R_{J}\right]\left[R_{K}, R_{I}\right] R_{J} R_{K}-4 c_{1}\left[R_{I}, R_{J}\right] R_{I}\left[R_{K}, R_{J}\right] R_{K}\right] .
\end{aligned}
$$

Here $R_{I}=U \partial_{I} U^{-1}$ is the right current and the Skyrme field is defined by the holonomy

$$
U\left(x^{I}\right)=-\mathrm{P} \exp \left[\int_{-\infty}^{\infty} d x^{8} A_{8}\left(x^{I}, x^{8}\right)\right]
$$

\footnotetext{
${ }^{4}$ In order that instantons are classified by the integer topological charge, it is necessary that the homotopy group contains at least one $\mathbb{Z}$ factor. For example, we can consider the gauge group $\mathrm{SO}(8)$ in that case we have $\pi_{7}(\mathrm{SO}(8))=\mathbb{Z} \times \mathbb{Z}$.
} 
Therefore, the Skyrme field is a map $U: \mathbb{R}^{7} \mapsto \tilde{G}$ where $\tilde{G}$ is a group manifold. The numerical constants $c_{1}, c_{2}$ in (2.15) are calculated to be

$$
c_{1}=\int_{-\infty}^{\infty} d x^{8} \frac{1}{2 \sqrt{\pi}} \psi_{0}^{2} \psi_{+}^{2}\left(\psi_{+}-1\right)^{2} \simeq 0.00940, \quad c_{2}=\int_{-\infty}^{\infty} d x^{8} \psi_{+}^{4}\left(\psi_{+}-1\right)^{4} \simeq 0.00308 .
$$

As in the case of the four-dimensional Skyrme model, these numerical factors are scaled away by the replacements $x^{I} \rightarrow \sqrt{c_{2} / c_{1}} x^{I}, E_{\text {Skyrme }} \rightarrow \frac{1}{\sqrt{c_{1} c_{2}}} E_{\text {Skyrme. }}$. We therefore set $c_{1}=c_{2}=1$. The quartic Yang-Mills action (2.14) has the scale invariance while the energy functional (2.15) does not. Again, this is due the Sutcliffe's truncation where only the zero-mode is considered and the vector mesons are neglected.

The eight-dimensional Skyrme model (2.15) has similar properties with the fourdimensional ones. For example, the energy functional (2.15) is invariant under the following global transformation

$$
U \rightarrow O_{L} U O_{R}^{-1}, \quad O_{L}, O_{R} \in \tilde{G} .
$$

This is a generalization of the chiral symmetry in four dimensions. One also finds that the energy functional (2.15) consists of the terms with 6th and 8th derivatives. This is compared with the 2nd and 4th derivative terms in the four-dimensional Skyrme model. The Derrick's theorem applied to the energy (2.15) indicates that there is a stable solitonic solution to this model. We call this the eight-dimensional Skyrmions. The Bogomol'nyi completion of the energy (2.15) is given by

$$
\begin{aligned}
& E_{\text {Skyrme }}=4 \int d^{7} x \operatorname{Tr}\left[\left(\sqrt{\frac{1}{3 !}} \varepsilon_{I J K L A B C} R_{I} R_{J} R_{K} \pm \sqrt{4 !} R_{[L} R_{A} R_{B} R_{C]}\right)^{2}\right. \\
&\left.\mp 4 \varepsilon_{I J K L A B C} R_{I} R_{J} R_{K} R_{L} R_{A} R_{B} R_{C}\right] \geq \frac{16}{N_{C}}|\mathcal{B}|,
\end{aligned}
$$

where $N_{C}=1 / 9600 \pi^{4}$ is the normalization constant of the following topological charge:

$$
\mathcal{B}=N_{C} \int d^{7} x \operatorname{Tr}\left[\varepsilon_{I J K L A B C} R_{I} R_{J} R_{K} R_{L} R_{A} R_{B} R_{C}\right] .
$$

Here $\varepsilon_{I J K L A B C}$ is the totally antisymmetric tensor. The topological charge (2.20) is the natural generalization of the Baryon number $B=-\frac{1}{24 \pi^{2}} \int d^{3} x \operatorname{Tr}\left[\varepsilon_{i j k} R_{i} R_{j} R_{k}\right]$ in the fourdimensional Skyrme model.

\section{Eight-dimensional Skyrmions from instantons}

In this section, we examine a field configuration that extremizes the energy functional (2.15), namely, the Skyrmion in eight dimensions. Assuming the hedgehog ansatz for the Skyrme field $U(x)$, we first derive the equation of motion from (2.15). We will find a solution to the equation by the numerical analysis. We then construct a field configuration from the eight-dimensional instantons through the Atiyah-Manton prescription. We compare the two solutions and verify whether the Atiyah-Manton approximation works even in eight dimensions. 
Skyrmions from numerical analysis. Following the standard scheme for a spherically symmetric solution to the four-dimensional Skyrme model, we consider the following hedgehog ansatz:

$$
U(x)=\exp \left(f(r) \hat{x}^{I} e_{I}^{\dagger}\right),
$$

where $\hat{x}^{I}=\frac{x^{I}}{r}, r^{2}=x^{I} x^{I}$ and $f(r)$ is a real function. The basis $e_{I}, e_{I}^{\dagger}$ is the higher dimensional analogue of the pure imaginary quaternions in four dimensions. Note that we do not employ the octonions as a higher dimensional generalisation of the quaternions. It is well known that the octonions are never represented by matrices and the algebra based on them loses the associativity [18]. The natural candidate for the basis in eight dimensions is based on the Clifford algebra [19]. This is given by

$$
e_{M}=\delta_{M 8} \mathbf{1}_{8}+\delta_{M I} \Gamma_{I}^{(-)}, \quad e_{M}^{\dagger}=\delta_{M 8} \mathbf{1}_{8}+\delta_{M I} \Gamma_{I}^{(+)}, \quad(M=1, \ldots, 8, I=1, \ldots, 7),
$$

where $\Gamma_{I}^{( \pm)}$are $8 \times 8$ matrices that satisfy the relations $\left\{\Gamma_{I}^{( \pm)}, \Gamma_{J}^{( \pm)}\right\}=-2 \delta_{I J} \mathbf{1}_{8}$. The matrices $\Gamma_{I}^{( \pm)}$are defined by $\Gamma_{I}^{( \pm)}=\frac{1}{2}(1 \pm \omega) \Gamma_{I}$. We choose the matrices $\Gamma_{I}^{( \pm)}$such that they satisfy the relation $\Gamma_{I}^{(+)}=-\Gamma_{I}^{(-)}$. Here $\Gamma_{I}$ are given by the matrix representation of the seven-dimensional complex Clifford algebra $\Gamma_{I} \in C \ell_{7}(\mathbb{C})$ and $\omega=(-1) \Gamma_{1} \cdots \Gamma_{7}$ is a chirality matrix. The basis is normalized as $\operatorname{Tr}\left[e_{M} e_{N}^{\dagger}\right]=8 \delta_{M N}$ and satisfies the following relations

$$
\begin{aligned}
& e_{M} e_{N}^{\dagger}+e_{N} e_{M}^{\dagger}=e_{M}^{\dagger} e_{N}+e_{N}^{\dagger} e_{M}=2 \delta_{M N} \mathbf{1}_{8}, \\
& e_{M} e_{N}+e_{N} e_{M}=2 \delta_{M 8} e_{N}+2 \delta_{N 8} e_{M}-2 \delta_{M N} \mathbf{1}_{8}, \\
& e_{M}^{\dagger} e_{N}^{\dagger}+e_{N}^{\dagger} e_{M}^{\dagger}=2 \delta_{M 8} e_{N}^{\dagger}+2 \delta_{N 8} e_{M}^{\dagger}-2 \delta_{M N} \mathbf{1}_{8} .
\end{aligned}
$$

Note that we have $e_{I}^{\dagger}=-e_{I}$ in our construction. Therefore the hedgehog field configuration (3.1) satisfies $U^{\dagger} U=\mathbf{1}_{8}$ and it belongs to $\mathrm{U}(8)$. The details of the Clifford algebra, including the explicit matrix representations of the basis $e_{M}, e_{M}^{\dagger}$, are found in [19].

We now derive the equation of motion for the profile function $f(r)$. Using the algebra of the basis (3.3), we find that the hedgehog ansatz is expanded as

$$
U(x)=\cos f \mathbf{1}_{8}+\sin f \hat{x}^{I} e_{I}^{\dagger} .
$$

This expression allows us to write down the right-current field:

$$
R_{I}=-r^{-1} \sin ^{2} f \hat{x}_{I} \mathbf{1}_{8}+\left(-r^{-1} \sin f \cos f+\partial_{r} f\right) \hat{x}_{I} \hat{x}^{\dagger}+r^{-1} \sin f \cos f e_{I}^{\dagger}-r^{-1} \sin ^{2} f e_{I}^{\dagger} \hat{x}^{\dagger} .
$$

Here $\hat{x}=\hat{x}^{I} e_{I}, \hat{x}^{\dagger}=\hat{x}^{I} e_{I}^{\dagger}$. It is straightforward to calculate each term in (2.15) by using the above expression and the algebra associated with the basis (3.3). Although the derivation is tedious, it needs a little bit of effort. The details are found in appendix. The energy functional becomes,

$$
\begin{aligned}
E_{\text {Skyrme }} & =\int_{0}^{\infty} d r \int_{S^{6}} d \Omega_{6} \mathcal{E}(r) \\
& =24576 \pi^{3} \int_{0}^{\infty} d r\left(3 r^{2} \sin ^{4} f\left(\partial_{r} f\right)^{2}+4 \sin ^{6} f\left(4\left(\partial_{r} f\right)^{2}+1\right)+12 \frac{\sin ^{8} f}{r^{2}}\right),
\end{aligned}
$$




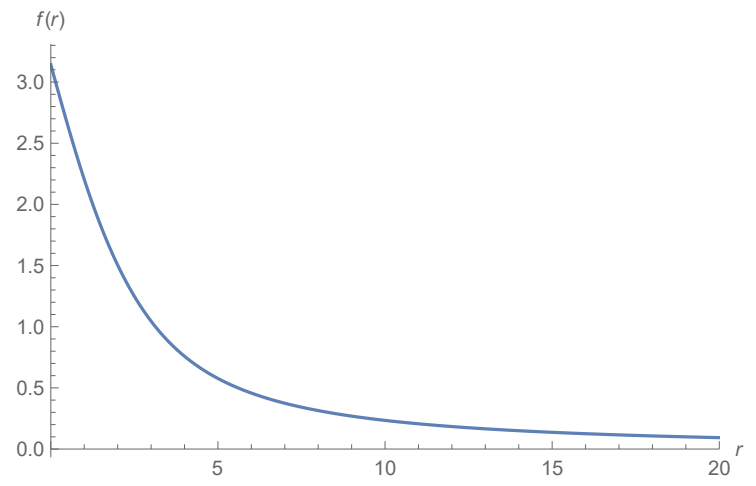

(a) The profile for the 8d Skyrmion.

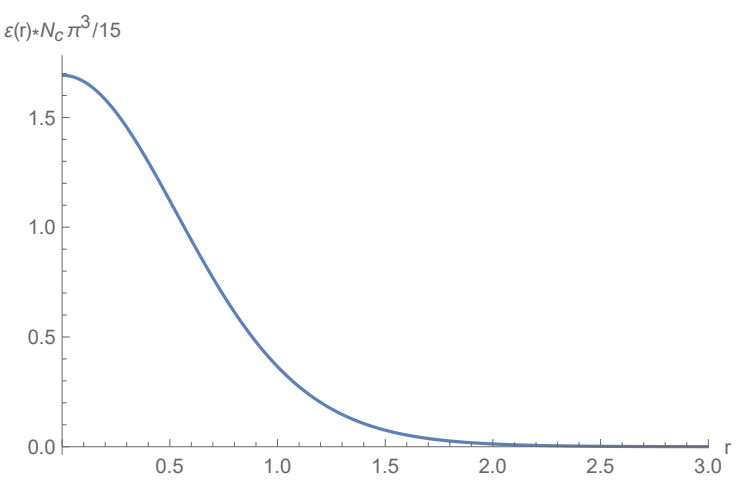

(b) The energy density plot for the $8 \mathrm{~d}$ Skyrmion.

Figure 2. The numerical profile for $f(r)$ and the plot for the energy density $\mathcal{E}(r)$.

where the overall factor comes from the volume factor of the radial direction and algebras containing $e_{M}, e_{M}^{\dagger}$ (see appendix). Then, we derive the equation of motion for $f(r)$ as

$$
\begin{gathered}
\sin ^{2} f\left(3 r^{2}+16 \sin ^{2} f\right) \partial_{r}^{2} f+6 r \sin ^{2} f \partial_{r} f \\
+3 \sin 2 f\left[\left(r^{2}+8 \sin ^{2} f\right)\left(\partial_{r} f\right)^{2}-2 \sin ^{2} f-8 \frac{\sin ^{4} f}{r^{2}}\right]=0 .
\end{gathered}
$$

The boundary condition for the profile function $f(r)$ is

$$
f(0)=\pi, \quad f(\infty)=0 .
$$

Compared with the equation in four dimensions, the equation (3.7) looks highly nonlinear. Therefore it is not obvious whether the equation (3.7) has appropriate solutions that are consistent with the boundary condition (3.8) or not. In order to clarify the existence of the solution to the equation (3.7), we first perform the Taylor expansion of the profile function at the origin: $f(\delta r)=\sum_{i=0}^{\infty} f_{i}(\delta r)^{i}=f_{0}+f_{1} \delta r+f_{2}(\delta r)^{2}+\ldots$ We then write down the equations for the coefficients $f_{i}$ and look for $f_{i}$ order by order in $(\delta r)^{i}$. For the boundary condition (3.8), we find that the asymptotic behavior of the solution at the origin is

$$
f(\delta r)=\pi+f_{1} \delta r-\frac{\left(3 c_{1}+8 c_{2} f_{1}^{2}\right) f_{1}^{3}}{9\left(3 c_{1}+16 c_{2} f_{1}^{2}\right)}(\delta r)^{3}+\mathcal{O}\left((\delta r)^{5}\right) .
$$

Here $f_{1}$ can be chosen as a shooting parameter in the numerical analysis. From this observation, we conclude that we can numerically calculate a solution to the equation (3.7) by appropriate methods of second ordinary differential equations with boundary conditions. We stress that, if any shooting parameters are not found, then the equation does not have appropriate solution with the boundary condition in general. The numerical result is found in figure 2 where we have employed the functional Newton-Raphson method. The behaviour of the profile function and the energy functional is quite similar to those in the four-dimensional Skyrmion (see figure 1).

The Skyrme field is a map $\mathbb{R}^{7} \mapsto \mathrm{U}(8)$. However, the boundary condition $U(r) \rightarrow \mathbf{1}_{8}$ $(r \rightarrow \infty)$ implies that the base manifold is topologically $S^{7}$. Therefore the solutions are 
characterized by the topological charge associated with the homotopy group $\pi_{7}(\mathrm{U}(8))=\mathbb{Z}$. Indeed, the topological charge for the hedgehog ansatz (3.1) and the boundary condition (3.8) is evaluated to be

$$
\mathcal{B}=-9600 \pi^{3} N_{C}(f(\infty)-f(0))=1
$$

This is the single Skyrmion in eight dimensions.

Atiyah-Manton solution from instantons. We next make contact with the Skyrmion from the eight-dimensional instantons. The Bogomol'nyi completion of the quartic YangMills action (2.14) is

$$
\begin{aligned}
S_{\text {quartic }} & =\int \operatorname{Tr}\left[\left(F \wedge F \mp *_{8}(F \wedge F)\right)^{2} \pm F \wedge F \wedge F \wedge F\right] \\
& \geq \pm \int \operatorname{Tr}[F \wedge F \wedge F \wedge F] .
\end{aligned}
$$

Here we have defined

$$
\left(F \wedge F \pm *_{8} F \wedge F\right)^{2}=\left(F \wedge F \pm *_{8} F \wedge F\right) \wedge *_{8}\left(F \wedge F \pm *_{8} F \wedge F\right)
$$

The action is bounded from below by the fourth Chern number $k=\int \operatorname{Tr}[F \wedge F \wedge F \wedge F]$ which defines the topological charge associated with instantons. The theory defined by the action (3.11) has scale invariance. The Derrick's theorem implies that the theory admits static solitons, namely, instantons. The Bogomol'nyi bound is saturated when the (anti-)self-duality equation

$$
F \wedge F= \pm *_{8} F \wedge F
$$

is satisfied. This is a natural generalization of the (anti-)self-duality equation $F= \pm *_{4}$ $F$ in four dimensions. In the following we choose the plus sign in (3.13). Solutions to the equation (3.13) is known as the self-dual instantons in eight dimensions. They are characterized by the homotopy group $\pi_{7}(G)=\mathbb{Z}$ where $G$ is a gauge group. Only the one-instanton is known as an analytic solution in the past $[15,16] .{ }^{5}$ The one-instanton solution is given by

$$
A_{M}=\frac{1}{4} \partial_{N} \ln \left(1+\frac{\lambda^{2}}{\|\tilde{x}\|^{2}}\right) \Sigma_{M N}^{(-)}
$$

where $\|\tilde{x}\|^{2}=\left(x^{M}-a^{M}\right)\left(x_{M}-a_{M}\right)$ and $\lambda, a^{M}$ are the size and the position moduli of the solution. For simplify, we set $a^{M}=0$. The matrix

$$
\Sigma_{M N}^{(-)}=e_{M} e_{N}^{\dagger}-e_{N} e_{M}^{\dagger}
$$

is the generator of the $\mathrm{SO}(8)$ Lorentz group. This is the eight-dimensional analogue of the 't Hooft instanton in four dimensions [20].

\footnotetext{
${ }^{5}$ We note that multi-instanton solutions to the self-duality equation (3.13) are discussed in the framework of the ADHM construction [19].
} 


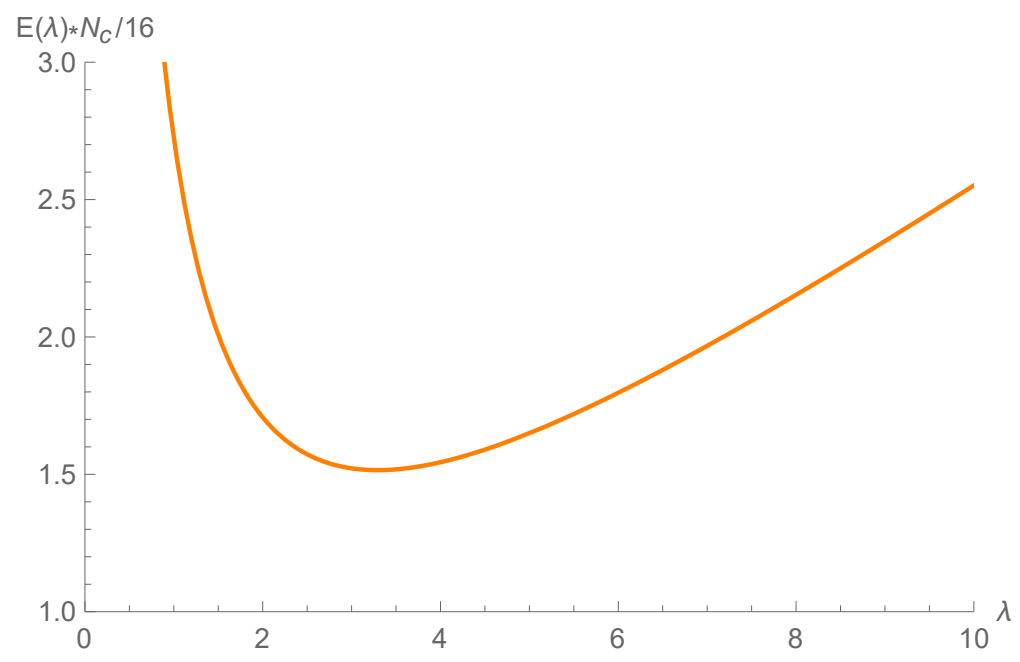

Figure 3. The energy profile for the Atiyah-Manton solution (3.18) as the function of the instanton size $\lambda$.

Following Atiyah and Manton [6], we calculate the holonomy for the instanton solution (3.14). To this end, it is convenient to rewrite the solution (3.14) as

$$
A_{M}\left(x^{I}, x^{8}\right)=\frac{1}{2}\left(\frac{1}{\lambda^{2}+r^{2}+\left(x^{8}\right)^{2}}-\frac{1}{r^{2}+\left(x^{8}\right)^{2}}\right) x^{N} \Sigma_{M N}^{(-)} .
$$

Then one finds

$$
A_{8}=\left(\frac{1}{\lambda^{2}+r^{2}+\left(x^{8}\right)^{2}}-\frac{1}{r^{2}+\left(x^{8}\right)^{2}}\right) x^{I} e_{I}^{\dagger} .
$$

Using this representation, we calculate the following holonomy for the one-instanton solution $A_{8}$ :

$$
U\left(x^{I}\right)=-\mathrm{P} \exp \int_{-\infty}^{\infty} d x^{8} A_{8}\left(x^{I}, x^{8}\right)=\exp \left[\pi\left(1-\frac{r}{\sqrt{r^{2}+\lambda^{2}}}\right) \hat{x}^{I} e_{I}^{\dagger}\right] .
$$

The result is the standard hedgehog form for the Skyrme field (3.1). This is why we have employed the basis $e_{I}^{\dagger}$ in (3.1). Plugging the Atiyah-Manton solution (3.18) into the quartic Yang-Mills action (2.14) results in the static energy $E(\lambda)$ for the solution. The plot for $E(\lambda)$ is found in figure 3. As anticipated, the energy depends on the size of the instanton $\lambda$. This is because the Sutcliffe's truncation breaks the scale invariance in the quartic YangMills model. The size $\lambda$ now lost its status of modulus. The true solution corresponds to the extremum of $E(\lambda)$. We find this happens at $\lambda=3.29095$.

For this value of $\lambda$, we now compare the profile functions of the Atiyah-Manton and the numerical solutions. The result is found in figure 4. We find that they agrees with high accuracy. The plot for the energy density is also compared in figure 5. Again, we find a good agreement between them. This result can be confirmed by evaluating the total energy (see table 1). We therefore conclude that the Atiyah-Manton construction of Skyrmions from instantons works well even in eight dimensions. We note that the eight-dimensional Skyrmion is a non-BPS solution which is same as the four-dimensional one. 


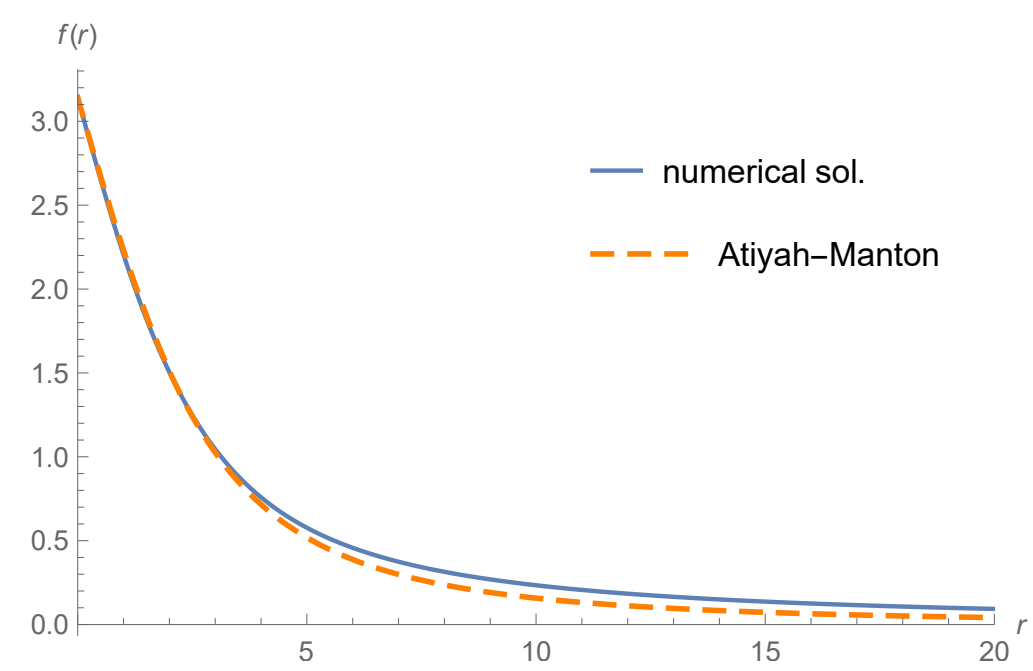

Figure 4. The profile functions for the numerical and the Atiyah-Manton solutions.

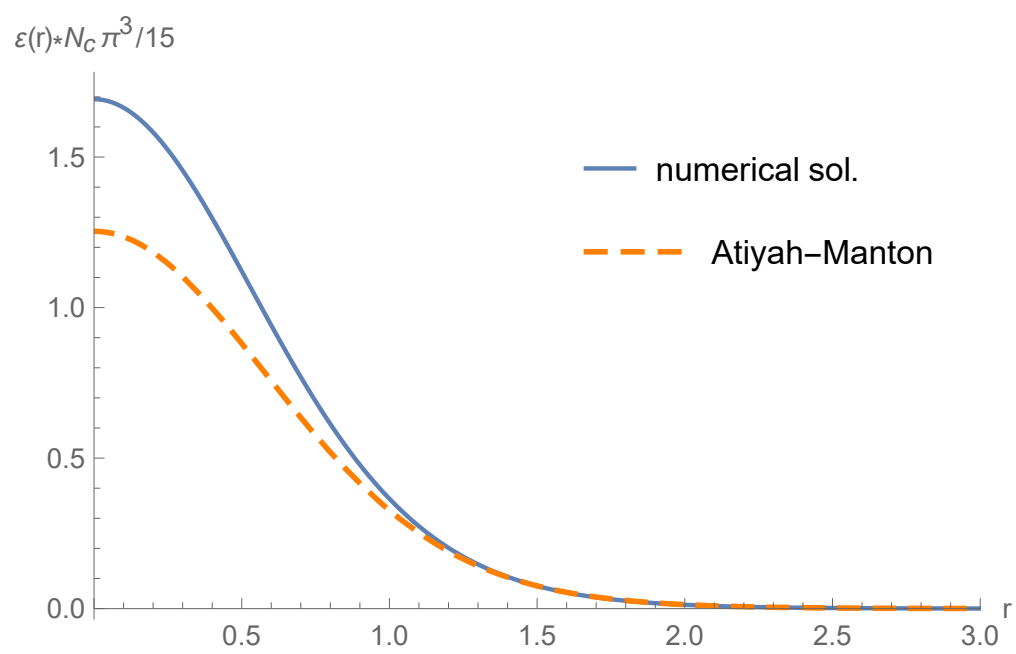

Figure 5. The profile functions for the energy density. The numerical versus the Atiyah-Manton solutions.

\begin{tabular}{|c|c|c|c|}
\hline Solution & Numerical & Atiyah-Manton & BPS bound \\
\hline Energy & $1.51239 \times 16 / N_{c}$ & $1.51521 \times 16 / N_{c}$ & $16 / N_{c}$ \\
\hline
\end{tabular}

Table 1. The total energy for the numerical, the Atiyah-Manton solutions and the BPS bound in this model (2.19).

\section{Higher dimensional generalization}

In this section we make an analysis on the Atiyah-Manton construction in $4 k$ dimensions. It is worthwhile first to mention about the $k=3$, namely, the twelve-dimensional case. In twelve dimensions, the self-duality equation becomes $F \wedge F \wedge F= \pm *_{12} F \wedge F \wedge F$. It is an easy exercise to show that the one-instanton solution to this equation is given by (3.14) 
where the $\mathrm{SO}(8)$ generator $\Sigma_{\mu \nu}^{(-)}$is replaced by that of $\mathrm{SO}(12)$. We can construct the AtiyahManton solution by calculating the holonomy associated with the instanton solution. We can also find the Skyrme model in twelve dimensions and its Skyrmion solution along the lines of the eight-dimensional case. The discussion is parallel to that in eight dimensions presented in this paper. However, the explicit calculation of the Sutcliffe's truncation in twelve dimensions results in the energy functional for the Skyrme model with diverse (about $\left.\mathcal{O}\left(10^{2}\right)\right)$ terms. Analyzing all the terms is beyond the scope of this paper. We therefore proceed to the general discussion in the following.

Now we move to the discussion in $4 k$ dimensions. The $4 k$-dimensional generalization of the quartic Yang-Mills action (2.14) is

$$
S_{\mathrm{YM}}=\frac{1}{2} \int_{\mathbb{R}^{4 k}} \operatorname{Tr}\left[\left(*_{4 k} F^{k}\right) \wedge\left(F^{k}\right)\right]
$$

where $F^{k}$ is the $k$ th wedge products of the gauge field strength 2 -form, $F^{k}=F \wedge \cdots \wedge F$. The gauge field takes value in the adjoint representation of a gauge group $G$. We assume that this gauge group has non-trivial homotopy $\pi_{4 k-1}(G)=\mathbb{Z}$. It is straightforward to perform the Bogomol'nyi completion of the action:

$$
S_{\mathrm{YM}}=\int_{\mathbb{R}^{4 k}} \operatorname{Tr}\left[\left(F^{k} \mp *_{4 k} F^{k}\right)^{2} \pm F^{4 k}\right] \geq \pm \int_{\mathbb{R}^{4 k}} \operatorname{Tr}\left[F^{4 k}\right] .
$$

The BPS equation becomes

$$
F^{k}= \pm *_{4 k} F^{k}
$$

This is the (anti-)self-duality equation in $4 k$ dimensions. The one-instanton solution to this equation is explicitly wrote down by the ADHM construction of instantons in $4 k$ dimensions [21] which is the $4 k$-dimensional generalization of [19] in eight dimensions. Again, the solutions are given as the form in (3.14) where the $\mathrm{SO}(8)$ generator is replaced by those of $\mathrm{SO}(4 k)$.

Next we perform the Sutcliffe's truncation. The index structure of the Yang-Mills Lagrangian is

$$
\begin{aligned}
\left(*_{4 k} F^{k}\right) & \wedge F^{k}= \\
& \frac{1}{(2 k) !}\left(\frac{1}{2 !}\right)^{2 k} \varepsilon_{M_{1} \cdots M_{2 k} N_{1} \cdots N_{2 k}} \varepsilon^{M_{1} \cdots M_{2 k} P_{1} \cdots P_{2 k}} F^{N_{1} N_{2}} \cdots F^{N_{2 k-1} N_{2 k}} F_{P_{1} P_{2}} \cdots F_{P_{2 k-1} P_{2 k}} d^{4 k} x,
\end{aligned}
$$

where the overall factor comes from the normalization of the 2-form $F=\frac{1}{2 !} F_{M N} d x^{M} \wedge d x^{N}$ and the definition of the Hodge dual operation. The procedure of the reduction is parallel to the previous sections. We can reduce the gauge field along, say, the $x^{4 k}$-direction. Then, the gauge field becomes

$$
\begin{gathered}
F_{I \sharp}=R_{I} \frac{\psi_{0}\left(x^{4 k}\right)}{\sqrt{2} \pi^{\frac{1}{4}}}, \quad F_{I J}=\left[R_{I}, R_{J}\right] \psi_{+}\left(x^{4 k}\right)\left(\psi_{+}\left(x^{4 k}\right)-1\right), \\
(I, J, \ldots=1, \ldots 4 k-1, \sharp=4 k) .
\end{gathered}
$$


Here $R_{I}=U \partial_{I} U^{\dagger}$ is the right current field constructed from the Skyrme field $U\left(x^{I}\right)$. Then, the energy functional for the static field $U\left(x^{I}\right)$ in $4 k$ dimensions has the following structure

$$
E_{\text {Skyrme }}=E_{4 k}(x)+E_{4 k-2}(x),
$$

where $E_{n}$ stands for terms that contain $n$-th derivatives. The energy (4.6) is compared with that in the eight-dimensional Skyrme model. Again, the Derrick's theorem implies that there is a static soliton solution that extremizes the energy (4.6). This is nothing but the Skyrmion in $4 k$ dimensions. Finding the explicit solutions need the numerical analysis in each dimension. We can also calculate the holonomy for the 1-instanton solution in $4 k$ dimensions and derive the static energy $E(\lambda)$. Although we do not repeat the same calculations, the result of the original Atiyah-Manton construction in four dimensions and our result in eight dimensions strongly suggest that this instanton/Skyrmion correspondence does hold in $4 k$ dimensions.

\section{Conclusion and discussions}

In this paper we studied the Atiyah-Manton construction of Skyrmions in eight dimensions. Following the formalism developed in [10], we derive the static energy functional for the Skyrme field from the quartic Yang-Mills action in eight dimensions. The Derrick's theorem indicates that there exist stable soliton solutions. The solutions are classified by the topological charge $\mathcal{B}=N_{C} \int d^{7} x \varepsilon_{I J K L A B C} \operatorname{Tr}\left[R_{I} R_{J} R_{K} R_{L} R_{A} R_{B} R_{C}\right]$ which is the eight-dimensional generalization of the Baryon number. Assuming the spherically symmetric hedgehog ansatz, we derive the equation of motion for the Skyrme field. Although the equation is quite nonlinear and complicated, we can perform the numerical analysis to find solutions. We present the explicit numerical solution for the Skyrmion associated with the topological charge $\mathcal{B}=1$. The profile function and the energy density of the eight-dimensional Skyrmion look quite similar to those in four dimensions.

In the latter part of the paper, we study the relation between the eight-dimensional instantons and the Skyrmions. This is a generalization of the Atiyah-Manton construction in four-dimensions. Following the four-dimensional case, we constructed the Atiyah-Manton solution for the Skyrmion from the one instanton solution found in $[15,16]$. We then compare the numerical solution and the Atiyah-Manton solution and find that there is a good agreement between them. The profile function looks quite similar in these solutions. This result dictates us that the correspondence between the instantons and the Skyrmions by the Atiyah-Manton construction is an universal property in higher dimensions.

Indeed, we have confirmed that the Sutcliffe's truncation of the higher dimensional generalization of the quartic Yang-Mills action gives the energy functional $E$ for the static Skyrme field in $4 k$ dimensions. The structure of $E$ together with the Derrick's theorem implies that there are stable Skyrmion solutions in $4 k$ dimensions. Since it is easy to show that the one-instantons in $4 k$ dimensions are given by the 't Hooft type, we can easily write down the Atiyah-Manton solution in each dimension. Although it is hard to compute the explicit energy functional for the $4 k$-dimensional Skyrme model, we expect the Atiyah-Manton solution provides a good approximation to the Skyrmions. 
Our study exhibits a deep relation between Yang-Mills instantons and Skyrmions. Physical interpretations of the Atiyah-Manton construction in lower dimensions are studied intensively [22-25]. Analogous relations among various solitons are expected in higher dimensions. Meanwhile, supersymmetry play an important role to study the BPS nature of classical solutions. Among other things, supersymmetric generalizations of Skyrme model in four dimensions have been studied [26-31]. It is interesting to supersymmetrize the eight dimensional Skyrme model presented in this paper.

There are various related studies. It is known that Skyrmions and monopoles have similar structures through the rational map ansatz. One can expect that this relation holds even in higher dimensions. For example, we know that only the numerical solutions of monopoles in seven dimensions [32]. It is interesting to study the Nahm construction of monopoles [33, 34] to find analytic solutions in seven dimensions. These expectations may be based on the integrable structure of the self-duality equations. It is known that the self-duality equation in four dimensions are reduced to integrable equations in lower dimensions [35]. It is also interesting to study the integrable structure of the self-duality equations in $4 k$ dimensions and generalization of the Ward's conjecture [36]. We will come back to these issues in future studies.

\section{Acknowledgments}

We would like to thank Muneto Nitta and Nobuyuki Sawado for useful discussions and comments. The work of S.S. is supported in part by Kitasato University Research Grant for Young Researchers.

\section{A Derivation of the eight-dimensional Skyrme model with the hedgehog ansatz}

For later convenience we reproduced the right current as

$$
R_{I}=-r^{-1} \sin ^{2} f \hat{x}_{I} \mathbf{1}_{8}+\left(-r^{-1} \sin f \cos f+\partial_{r} f\right) \hat{x}_{I} \hat{x}^{\dagger}+r^{-1} \sin f \cos f e_{I}^{\dagger}-r^{-1} \sin ^{2} f e_{I}^{\dagger} \hat{x}^{\dagger} .
$$

Then we find

$$
R_{I} R_{I}=-\left(\left(\partial_{r} f\right)^{2}+6 r^{-2} \sin ^{2} f\right) \mathbf{1}_{8} .
$$

The commutator of the current $R_{I}$ is calculated to be

$$
\begin{aligned}
{\left[R_{I}, R_{J}\right]=} & -r^{-2} \sin ^{2} f \Sigma_{I J}^{(-)} \\
& +2\left(r^{-2} \sin ^{2} f-r^{-1} \sin f \cos f \partial_{r} f\right)\left(\hat{x}_{I} e_{J}^{\dagger}-\hat{x}_{J} e_{I}^{\dagger}\right) \hat{x}^{\dagger}-2 r^{-1} \sin ^{2} f \partial_{r} f\left(\hat{x}_{I} e_{J}^{\dagger}-\hat{x}_{J} e_{I}^{\dagger}\right) \\
= & -D \Sigma_{I J}^{(-)}+E \Theta_{I J} \hat{x}^{\dagger}-F \Theta_{I J},
\end{aligned}
$$

where we have defined $D=r^{-2} \sin ^{2} f, E=2\left(r^{-2} \sin ^{2} f-r^{-1} \sin f \cos f \partial_{r} f\right), \quad F=$ $2 r^{-1} \sin ^{2} f \partial_{r} f$ and $\Theta_{I J}=\hat{x}_{I} e_{J}^{\dagger}-\hat{x}_{J} e_{I}^{\dagger}$. Here the matrices $\Sigma_{I J}^{(-)}$and $\Theta_{I J}$ satisfy the following relations

$$
\begin{aligned}
& \Theta_{I J} \hat{x}^{\dagger}=-\hat{x}^{\dagger} \Theta_{I J}, \quad \Sigma_{I J}^{(-)} \Theta_{I J}=4 \cdot 6 \hat{x}^{\dagger}, \quad \Theta_{I J} \Sigma_{I J}^{(-)}=-4 \cdot 6 \hat{x}^{\dagger}, \\
& \Theta_{I J}^{2}=-2 \cdot 6 \mathbf{1}_{8}, \quad\left(\Sigma_{I J}^{(-)}\right)^{2}=-4 \cdot 7 \cdot 6 \mathbf{1}_{8} .
\end{aligned}
$$


The squares of the commutator $\left[R_{I}, R_{J}\right]$ is evaluated as

$$
\left[R_{I}, R_{J}\right]^{2}=-24 r^{-2} \sin ^{2} f\left(5 r^{-2} \sin ^{2} f+2\left(\partial_{r} f\right)^{2}\right) \mathbf{1}_{8}
$$

Using this result, we can calculate the first term in (2.15) as

$$
\left(\left[R_{I}, R_{J}\right]^{2}\right)^{2}=16 \cdot 6^{2} r^{-4} \sin ^{4} f\left(25 r^{-4} \sin ^{4} f+20 r^{-2} \sin ^{2} f\left(\partial_{r} f\right)^{2}+4\left(\partial_{r} f\right)^{4}\right) \mathbf{1}_{8}
$$

Things get more involved when we calculate the second term. We expand the second term in $(2.15)$ as

$$
\begin{aligned}
\left(\left[R_{I},\right.\right. & \left.\left.R_{J}\right]\left[R_{K}, R_{L}\right]\right)^{2}= \\
& D^{4} \Sigma_{I J}^{(-)} \Sigma_{K L}^{(-)} \Sigma_{I J}^{(-)} \Sigma_{K L}^{(-)} \\
& -D^{3} E\left(\Sigma_{I J}^{(-)} \Sigma_{K L}^{(-)} \Sigma_{I J}^{(-)} \Theta_{K L} \hat{x}^{\dagger}+\Sigma_{I J}^{(-)} \Sigma_{K L}^{(-)} \Theta_{I J} \hat{x}^{\dagger} \Sigma_{K L}^{(-)}+\Sigma_{I J}^{(-)} \Theta_{K L} \hat{x}^{\dagger} \Sigma_{I J}^{(-)} \Sigma_{K L}^{(-)}+\Theta_{I J} \hat{x}^{\dagger} \Sigma_{K L}^{(-)} \Sigma_{I J}^{(-)} \Sigma_{K L}^{(-)}\right) \\
+ & D^{3} F\left(\Sigma_{I J}^{(-)} \Sigma_{K L}^{(-)} \Sigma_{I J}^{(-)} \Theta_{K L}+\Sigma_{I J}^{(-)} \Sigma_{K L}^{(-)} \Theta_{I J} \Sigma_{K L}^{(-)}+\Sigma_{I J}^{(-)} \Theta_{K L} \Sigma_{I J}^{(-)} \Sigma_{K L}^{(-)}+\Theta_{I J} \Sigma_{K L}^{(-)} \Sigma_{I J}^{(-)} \Sigma_{K L}^{(-)}\right) \\
+ & D^{2}\left(E^{2}+F^{2}\right)\left(\Sigma_{I J}^{(-)} \Sigma_{K L}^{(-)} \Theta_{I J} \Theta_{K L}+\Theta_{I J} \Theta_{K L} \Sigma_{I J}^{(-)} \Sigma_{K L}^{(-)}\right) \\
+ & D^{2} E^{2}\left(\Sigma_{I J}^{(-)} \Theta_{K L} \hat{x}^{\dagger} \Sigma_{I J}^{(-)} \Theta_{K L} \hat{x}^{\dagger}+\Sigma_{I J}^{(-)} \Theta_{K L} \hat{x}^{\dagger} \Theta_{I J} \hat{x}^{\dagger} \Sigma_{K L}^{(-)}\right. \\
& \left.\quad+\Theta_{I J} \hat{x}^{\dagger} \Sigma_{K L}^{(-)} \Sigma_{I J}^{(-)} \Theta_{K L} \hat{x}^{\dagger}+\Theta_{I J} \hat{x}^{\dagger} \Sigma_{K L}^{(-)} \Theta_{I J} \hat{x}^{\dagger} \Sigma_{K L}^{(-)}\right) \\
- & D^{2} E F\left(\Sigma_{I J}^{(-)} \Theta_{K L} \hat{x}^{\dagger} \Sigma_{I J}^{(-)} \Theta_{K L}+\Sigma_{I J}^{(-)} \Theta_{K L} \hat{x}^{\dagger} \Theta_{I J} \Sigma_{K L}^{(-)}+\Theta_{I J} \hat{x}^{\dagger} \Sigma_{K L}^{(-)} \Sigma_{I J}^{(-)} \Theta_{K L}+\Theta_{I J} \hat{x}^{\dagger} \Sigma_{K L}^{(-)} \Theta_{I J} \Sigma_{K L}^{(-)}\right. \\
& \left.\quad+\Sigma_{I J}^{(-)} \Theta_{K L} \Sigma_{I J}^{(-)} \Theta_{K L} \hat{x}^{\dagger}+\Sigma_{I J}^{(-)} \Theta_{K L} \Theta_{I J} \hat{x}^{\dagger} \Sigma_{K L}^{(-)}+\Theta_{I J} \Sigma_{K L}^{(-)} \Sigma_{I J}^{(-)} \Theta_{K L} \hat{x}^{\dagger}+\Theta_{I J} \Sigma_{K L}^{(-)} \Theta_{I J} \hat{x}^{\dagger} \Sigma_{K L}^{(-)}\right) \\
& -D E\left(E^{2}+F^{2}\right)\left(\Sigma_{I J}^{(-)} \Theta_{K L} \hat{x}^{\dagger} \Theta_{I J} \Theta_{K L}+\Theta_{I J} \hat{x}^{\dagger} \Sigma_{K L}^{(-)} \Theta_{I J} \Theta_{K L}\right. \\
& \left.\quad+\Theta_{I J} \Theta_{K L} \Sigma_{I J}^{(-)} \Theta_{K L} \hat{x}^{\dagger}+\Theta_{I J} \Theta_{K L} \Theta_{I J} \hat{x}^{\dagger} \Sigma_{K L}^{(-)}\right) \\
+ & D^{2} F^{2}\left(\Sigma_{I J}^{(-)} \Theta_{K L} \Sigma_{I J}^{(-)} \Theta_{K L}+\Sigma_{I J}^{(-)} \Theta_{K L} \Theta_{I J} \Sigma_{K L}^{(-)}+\Theta_{I J} \Sigma_{K L}^{(-)} \Sigma_{I J}^{(-)} \Theta_{K L}+\Theta_{I J} \Sigma_{K L}^{(-)} \Theta_{I J} \Sigma_{K L}^{(-)}\right) \\
+ & D F\left(E^{2}+F^{2}\right)\left(\Sigma_{I J}^{(-)} \Theta_{K L} \Theta_{I J} \Theta_{K L}+\Theta_{I J} \Sigma_{K L}^{(-)} \Theta_{I J} \Theta_{K L}\right. \\
& \left.\quad+\Theta_{I J} \Theta_{K L} \Sigma_{I J}^{(-)} \Theta_{K L}+\Theta_{I J} \Theta_{K L} \Theta_{I J} \Sigma_{K L}^{(-)}\right) \\
+ & \left(E^{2}+F^{2}\right)^{2} \Theta_{I J} \Theta_{K L} \Theta_{I J} \Theta_{K L} .
\end{aligned}
$$

Here we have used the relation $\Theta_{I J} \hat{x}^{\dagger} \Theta_{K L} \hat{x}^{\dagger}=\Theta_{I J} \Theta_{K L}$ and $\Theta_{I J} \hat{x}^{\dagger} \Theta_{K L}+\Theta_{I J} \Theta_{K L} \hat{x}^{\dagger}=0$. We stress that terms that contain the odd number of $\hat{x}$ or $\hat{x}^{\dagger}$ vanish under the trace of the matrices. Since we need the trace of (A.7) in the energy functional, we neglect these terms and never calculate them in the following. Exploiting this fact, we are left with the terms 
that contain the even number of $\hat{x}$ :

$$
\begin{aligned}
& D^{4} \text { term: } \\
& \Sigma_{I J}^{(-)} \Sigma_{K L}^{(-)} \Sigma_{I J}^{(-)} \Sigma_{K L}^{(-)}=1344 \mathbf{1}_{8} \\
& D^{3} E \text { term: } \quad \Sigma_{I J}^{(-)} \Sigma_{K L}^{(-)} \Sigma_{I J}^{(-)} \Theta_{K L} \hat{x}^{\dagger}+\cdots+\Theta_{I J} \hat{x}^{\dagger} \Sigma_{K L}^{(-)} \Sigma_{I J}^{(-)} \Sigma_{K L}^{(-)}=4 \cdot 192 \mathbf{1}_{8}, \\
& D^{2}\left(E^{2}+F^{2}\right) \text { term: } \\
& \Sigma_{I J}^{(-)} \Sigma_{K L}^{(-)} \Theta_{I J} \Theta_{K L}+\Theta_{I J} \Theta_{K L} \Sigma_{I J}^{(-)} \Sigma_{K L}^{(-)}=-2 \cdot 384 \mathbf{1}_{8}, \\
& D^{2} E^{2} \text { term: } \quad \Sigma_{I J}^{(-)} \Theta_{K L} \hat{x}^{\dagger} \Sigma_{I J}^{(-)} \Theta_{K L} \hat{x}^{\dagger}+\Theta_{I J} \hat{x}^{\dagger} \Sigma_{K L}^{(-)} \Theta_{I J} \hat{x}^{\dagger} \Sigma_{K L}^{(-)}=2 \cdot 96 \mathbf{1}_{8}, \\
& \Sigma_{I J}^{(-)} \Theta_{K L} \hat{x}^{\dagger} \Theta_{I J} \hat{x}^{\dagger} \Sigma_{K L}^{(-)}+\Theta_{I J} \hat{x}^{\dagger} \Sigma_{K L}^{(-)} \Sigma_{I J}^{(-)} \Theta_{K L} \hat{x}^{\dagger}=-2 \cdot 384 \mathbf{1}_{8}, \\
& D E\left(E^{2}+F^{2}\right) \text { term: } \quad \Sigma_{I J}^{(-)} \Theta_{K L} \hat{x}^{\dagger} \Theta_{I J} \Theta_{K L}+\cdots+\Theta_{I J} \Theta_{K L} \Theta_{I J} \hat{x}^{\dagger} \Sigma_{K L}^{(-)}=-4 \cdot 192 \mathbf{1}_{8} \text {, } \\
& D^{2} F^{2} \text { term: } \quad \Sigma_{I J}^{(-)} \Theta_{K L} \Sigma_{I J}^{(-)} \Theta_{K L}+\Theta_{I J} \Sigma_{K L}^{(-)} \Theta_{I J} \Sigma_{K L}^{(-)}=2 \cdot 864 \mathbf{1}_{8} \text {, } \\
& \left(E^{2}+F^{2}\right)^{2} \text { term: } \\
& \Sigma_{I J}^{(-)} \Theta_{K L} \Theta_{I J} \Sigma_{K L}^{(-)}+\Theta_{I J} \Sigma_{K L}^{(-)} \Sigma_{I J}^{(-)} \Theta_{K L}=-2 \cdot 384 \mathbf{1}_{8}, \\
& \Theta_{I J} \Theta_{K L} \Theta_{I J} \Theta_{K L}=-96 \mathbf{1}_{8} \text {. }
\end{aligned}
$$

With this result at hand, we find that the second term in (2.15) becomes

$$
\operatorname{Tr}\left(\left[R_{I}, R_{J}\right]\left[R_{K}, R_{l}\right]\right)^{2}=1536 r^{-4} \sin ^{4} f\left(-5 r^{-4} \sin ^{4} f+20 r^{-2} \sin ^{2} f\left(\partial_{r} f\right)^{2}-8\left(\partial_{r} f\right)^{4}\right) .
$$

We can calculate the other terms by same method. After the calculations, the results are

$$
\begin{aligned}
\operatorname{Tr}\left(\left[R_{I}, R_{J}\right]^{2}\right)^{2} & =4608 r^{-4} \sin ^{4} f\left(25 r^{-4} \sin ^{4} f+20 r^{-2} \sin ^{2} f\left(\partial_{r} f\right)^{2}+4\left(\partial_{r} f\right)^{4}\right), \\
\operatorname{Tr}\left(\left[R_{I}, R_{J}\right]\left[R_{K}, R_{l}\right]\right)^{2} & =1536 r^{-4} \sin ^{4} f\left(-5 r^{-4} \sin ^{4} f+20 r^{-2} \sin ^{2} f\left(\partial_{r} f\right)^{2}-8\left(\partial_{r} f\right)^{4}\right), \\
\operatorname{Tr}\left[R_{I}, R_{J}\right]\left[R_{K}, R_{l}\right]\left[R_{I}, R_{K}\right]\left[R_{J}, R_{l}\right] & =768 r^{-4} \sin ^{4} f\left(-55 r^{-4} \sin ^{4} f-80 r^{-2} \sin ^{2} f\left(\partial_{r} f\right)^{2}+2\left(\partial_{r} f\right)^{4}\right), \\
\operatorname{Tr}\left(\left[R_{I}, R_{J}\right]\right)^{2} R_{K}^{2} & =192 r^{-2} \sin ^{2} f\left(30 r^{-4} \sin ^{4} f+17 r^{-2} \sin ^{2} f\left(\partial_{r} f\right)^{2}+2\left(\partial_{r} f\right)^{4}\right), \\
\operatorname{Tr}\left(\left[R_{I}, R_{J}\right] R_{K}\right)^{2} & =192 r^{-2} \sin ^{2} f\left(10 r^{-4} \sin ^{4} f+13 r^{-2} \sin ^{2} f\left(\partial_{r} f\right)^{2}-2\left(\partial_{r} f\right)^{4}\right), \\
\operatorname{Tr}\left[R_{I}, R_{J}\right] R_{K}\left[R_{I}, R_{K}\right] R_{J} & =192 r^{-2} \sin ^{2} f\left(-25 r^{-4} \sin ^{4} f-16 r^{-2} \sin ^{2}\left(\partial_{r} f\right)^{2}-\left(\partial_{r} f\right)^{4}\right), \\
\operatorname{Tr}\left[R_{I}, R_{J}\right]\left[R_{K}, R_{I}\right] R_{J} R_{K} & =192 r^{-2} \sin ^{2} f\left(15 r^{-4} \sin ^{4} f+14 r^{-2} \sin ^{2} f\left(\partial_{r} f\right)^{2}-\left(\partial_{r} f\right)^{4}\right), \\
\operatorname{Tr}\left[R_{I}, R_{J}\right] R_{I}\left[R_{K}, R_{J}\right] R_{K} & =192 r^{-2} \sin ^{2} f\left(-25 r^{-4} \sin ^{4} f-16 r^{-2} \sin ^{2}\left(\partial_{r} f\right)^{2}-\left(\partial_{r} f\right)^{4}\right) .
\end{aligned}
$$

Collecting everything altogether, we finally obtain

$$
\begin{gathered}
\operatorname{Tr}\left[c_{2}\left(\left[R_{I}, R_{J}\right]\left[R_{I}, R_{J}\right]\right)^{2}+c_{2}\left(\left[R_{I}, R_{J}\right]\left[R_{K}, R_{L}\right]\right)^{2}-4 c_{2}\left[R_{I}, R_{J}\right]\left[R_{K}, R_{L}\right]\left[R_{I}, R_{K}\right]\left[R_{J}, R_{L}\right]\right. \\
+4 c_{1}\left(\left[R_{I}, R_{J}\right]\right)^{2} R_{K}^{2}+4 c_{1}\left(\left[R_{I}, R_{J}\right] R_{K}\right)^{2}-4 c_{1}\left[R_{I}, R_{J}\right] R_{K}\left[R_{I}, R_{K}\right] R_{J} \\
\left.+8 c_{1}\left[R_{I}, R_{J}\right]\left[R_{K}, R_{I}\right] R_{J} R_{K}-4 c_{1}\left[R_{I}, R_{J}\right] R_{I}\left[R_{K}, R_{J}\right] R_{K}\right]= \\
23040\left(3 c_{1} r^{-4} \sin ^{4} f\left(\partial_{r} f\right)^{2}+4 r^{-6} \sin ^{6} f\left(4 c_{2}\left(\partial_{r} f\right)^{2}+c_{1}\right)+12 c_{2} r^{-8} \sin ^{8} f\right) .
\end{gathered}
$$

Taking $c_{1}=c_{2}=1$ and introducing the overall factor $\frac{16}{15} \pi^{3} r^{6}$, we obtain the energy functional (3.6). Here we have taken into account the factor that comes from the sixdimensional spherical integration:

$$
\int_{S^{6}} d \Omega_{6}=\frac{16}{15} \pi^{3} r^{6}
$$


where $S^{6}$ is the six-dimensional spherical surface and $d \Omega_{6}$ is the integral element of the six-dimensional sphere.

Open Access. This article is distributed under the terms of the Creative Commons Attribution License (CC-BY 4.0), which permits any use, distribution and reproduction in any medium, provided the original author(s) and source are credited.

\section{References}

[1] T.H.R. Skyrme, A unified field theory of mesons and baryons, Nucl. Phys. 31 (1962) 556 [INSPIRE].

[2] N.S. Manton and P.J. Ruback, Skyrmions in flat space and curved space, Phys. Lett. B 181 (1986) 137 [INSPIRE].

[3] N.S. Manton, Geometry of Skyrmions, Commun. Math. Phys. 111 (1987) 469 [InSPIRE].

[4] F. Canfora, F. Correa and J. Zanelli, Exact multisoliton solutions in the four-dimensional Skyrme model, Phys. Rev. D 90 (2014) 085002 [arXiv:1406.4136] [INSPIRE].

[5] C.J. Houghton, N.S. Manton and P.M. Sutcliffe, Rational maps, monopoles and Skyrmions, Nucl. Phys. B 510 (1998) 507 [hep-th/9705151] [InSPIRE].

[6] M.F. Atiyah and N.S. Manton, Skyrmions from instantons, Phys. Lett. B 222 (1989) 438 [INSPIRE].

[7] N.S. Manton and T.M. Samols, Skyrmions on $S^{3}$ and $H^{3}$ from instantons, J. Phys. A 23 (1990) 3749 [inSPIRE].

[8] M. Eto, M. Nitta, K. Ohashi and D. Tong, Skyrmions from instantons inside domain walls, Phys. Rev. Lett. 95 (2005) 252003 [hep-th/0508130] [InSPIRE].

[9] H. Hata, T. Sakai, S. Sugimoto and S. Yamato, Baryons from instantons in holographic QCD, Prog. Theor. Phys. 117 (2007) 1157 [hep-th/0701280] [InSPIRE].

[10] P. Sutcliffe, Skyrmions, instantons and holography, JHEP 08 (2010) 019 [arXiv:1003.0023] [INSPIRE].

[11] T. Sakai and S. Sugimoto, Low energy hadron physics in holographic QCD, Prog. Theor. Phys. 113 (2005) 843 [hep-th/0412141] [INSPIRE].

[12] P. Sutcliffe, Holographic Skyrmions, Mod. Phys. Lett. B 29 (2015) 1540051 [InSPIRE].

[13] P.M. Sutcliffe, Sine-Gordon solitions from CP $P^{1}$ instantons, Phys. Lett. B 283 (1992) 85 [INSPIRE].

[14] G.N. Stratopoulos and W.J. Zakrzewski, Approximate sine-Gordon solitons, Z. Phys. C 59 (1993) 307 [INSPIRE].

[15] B. Grossman, T.W. Kephart and J.D. Stasheff, Solutions to Yang-Mills field equations in eight-dimensions and the last Hopf map, Commun. Math. Phys. 96 (1984) 431 [Erratum ibid. 100 (1985) 311] [INSPIRE].

[16] D.H. Tchrakian, Spherically symmetric gauge field configurations with finite action in $4 p$-dimensions ( $p=$ integer), Phys. Lett. B 150 (1985) 360 [INSPIRE].

[17] M. Nitta, Matryoshka Skyrmions, Nucl. Phys. B 872 (2013) 62 [arXiv:1211.4916] [INSPIRE]. 
[18] J.C. Baez, The octonions, Bull. Am. Math. Soc. 39 (2002) 145 [math.RA/0105155] [inSPIRE].

[19] A. Nakamula, S. Sasaki and K. Takesue, ADHM construction of (anti-)self-dual instantons in eight dimensions, Nucl. Phys. B 910 (2016) 199 [arXiv:1604.01893] [INSPIRE].

[20] G. 't Hooft, Computation of the quantum effects due to a four-dimensional pseudoparticle, Phys. Rev. D 14 (1976) 3432 [Erratum ibid. D 18 (1978) 2199] [INSPIRE].

[21] K. Takesue, ADHM construction of (anti-)self-dual instantons in $4 n$ dimensions, work in progress.

[22] A.E. Kudryavtsev, B.M.A.G. Piette and W.J. Zakrzewski, Skyrmions and domain walls in (2+1)-dimensions, Nonlinearity 11 (1998) 783 [hep-th/9709187] [INSPIRE].

[23] M. Nitta, Josephson vortices and the Atiyah-Manton construction, Phys. Rev. D 86 (2012) 125004 [arXiv:1207.6958] [InSPIRE].

[24] M. Nitta, Correspondence between Skyrmions in 2+1 and $3+1$ dimensions, Phys. Rev. D 87 (2013) 025013 [arXiv:1210.2233] [INSPIRE].

[25] M. Eto and M. Nitta, Non-Abelian sine-Gordon solitons: correspondence between $\mathrm{SU}(N)$ Skyrmions and $\mathbb{C} P^{N-1}$ lumps, Phys. Rev. D 91 (2015) 085044 [arXiv:1501.07038] [INSPIRE].

[26] E.A. Bergshoeff, R.I. Nepomechie and H.J. Schnitzer, Supersymmetric Skyrmions in four-dimensions, Nucl. Phys. B 249 (1985) 93 [INSPIRE].

[27] L. Freyhult, The supersymmetric extension of the Faddeev model, Nucl. Phys. B 681 (2004) 65 [hep-th/0310261] [INSPIRE].

[28] C. Adam, J.M. Queiruga, J. Sanchez-Guillen and A. Wereszczynski, Extended supersymmetry and BPS solutions in baby Skyrme models, JHEP 05 (2013) 108 [arXiv:1304.0774] [INSPIRE].

[29] J.M. Queiruga, Skyrme-like models and supersymmetry in 3+1 dimensions, Phys. Rev. D 92 (2015) 105012 [arXiv: 1508.06692] [InSPIRE].

[30] S.B. Gudnason, M. Nitta and S. Sasaki, A supersymmetric Skyrme model, JHEP 02 (2016) 074 [arXiv: 1512.07557] [INSPIRE].

[31] S.B. Gudnason, M. Nitta and S. Sasaki, Topological solitons in the supersymmetric Skyrme model, JHEP 01 (2017) 014 [arXiv:1608.03526] [INSPIRE].

[32] E. Radu and D.H. Tchrakian, Static BPS 'monopoles' in all even spacetime dimensions, Phys. Rev. D 71 (2005) 125013 [hep-th/0502025] [InSPIRE].

[33] W. Nahm, A simple formalism for the BPS monopole, Phys. Lett. B 90 (1980) 413 [INSPIRE].

[34] W. Nahm, On Abelian self-dual multimonopoles, Phys. Lett. B 93 (1980) 42 [INSPIRE].

[35] L.J. Mason and N.M.J. Woodhouse, Integrability, selfduality, and twistor theory, London Mathematical Society Monographs, New Series: 15, Clarendon, Oxford U.K. (1996) [INSPIRE].

[36] R.S. Ward and M. Tabor, Integrable and solvable systems and relations among them, Phil. Trans. Roy. Soc. Lond. A 315 (1985) 451 [InSPIRE]. 